\title{
Influência da luz sobre o crescimento e a produção de biomassa de Ruppia maritima L. em cultivo experimental
}

\author{
Ioni Gonçalves Colares ${ }^{1,3}$ e Ulrich Seeliger ${ }^{2}$
}

Recebido em 5/12/2003. Aceito em 17/06/2005.

\begin{abstract}
RESUMO - (Influência da luz sobre o crescimento e a produção de biomassa de Ruppia maritima L. em cultivo experimental). O efeito de diferentes regimes de luz sobre o crescimento de Ruppia marítima foi avaliado nos níveis de irradiância de 40, 200 e $400 \mu$ mol $\mathrm{m}^{-2} \mathrm{~s}^{-1}$, durante 40 dias. A longevidade de novas folhas produzidas foi determinada e a formação de folhas, raízes e "ramets" registrada diariamente. As respostas de crescimento de $R$. maritima variaram em função dos níveis de irradiância, durante as condições de cultivo experimental, com as plantas apresentando diferentes estratégias de adaptação aos diferentes regimes de luz testados. Em irradiância de $40 \mu \mathrm{mol} \mathrm{m} \mathrm{m}^{-2} \mathrm{~s}^{-1}$, a longevidade $(57,7 \pm 2,7$ dias $)$ e o comprimento final das folhas $(11,5 \pm 0,3 \mathrm{~cm})$ foram maiores, embora a biomassa aérea e a taxa de crescimento específico das folhas tenham sido menores. Maiores irradiâncias levaram à maior produção de folhas, raízes, "ramets" e ramificações. Os resultados obtidos mostram que $R$. maritima apresenta melhor crescimento em ambientes com maiores intensidades luminosas e possui capacidade de ajustar suas características demográficas e respostas de crescimento a diferentes condições de luminosidade do ambiente
\end{abstract}

Palavras-chave : Ruppia maritima, crescimento, biomassa, longevidade das folhas, intensidade luminosa.

\begin{abstract}
Influence of light regimes on growth and biomass production of Ruppia maritima L. under controlled culture conditions). The effect of different light regimes on growth of plants of Ruppia maritima plants was assessed at irradiance levels of 40, 200 and $400 \mu \mathrm{mol} \mathrm{m}^{-2} \mathrm{~s}^{-1}$, for 40 days. The longevity of newborn leaves was determined and the formation of leaves, roots and ramets was recorded daily. The growth response of $R$. maritima varied as a function of irradiance levels during experimental culture conditions, and plants showed different strategies of adapting to the different light regimes tested. At irradiance of $40 \mu \mathrm{mol} \mathrm{m}^{-2} \mathrm{~s}^{-1}$, longevity $(57.7 \pm 2.7$ days) and final leaf length $(11.5 \pm 0.3 \mathrm{~cm})$ were greater, but aerial biomass and specific growth rate of the leaves were lower. Higher levels of irradiance caused higher production of leaves, roots, ramets and ramifications. The results showed that $R$. maritima grows better at higher light intensities, and can adjust demographic characteristics and growth to different light conditions in the environment.
\end{abstract}

Key words: Ruppia maritima, growth, biomass, leaf longevity, light intensity

\section{Introdução}

Fanerógamas submersas de regiões temperadas apresentam marcante sazonalidade no crescimento e na produção de biomassa em resposta as variações nas condições ambientais de luz, temperatura, salinidade e outros fatores abióticos (Duarte 1991a). Variações sazonais da irradiância subaquática podem levar a alteração na taxa de produção e alongamento das folhas, no comprimento dos rizomas, no número de ramificações e na produção de raízes (Duarte 1991b; Menéndez 2002). A redução na irradiância subaquática pode levar a uma diminuição na densidade de folhas e na biomassa de fanerógamas submersas, $o$ que atenua o alto sombreamento, permitindo às plantas um prolongamento do período de crescimento e uma expansão de seus limites de profundidade (Olesen \& Sand-Jensen 1993; Carruthers et al. 1999). O aumento do comprimento das folhas, em resposta à redução na irradiância subaquática, resulta em aumento da área foliar e maior superfície para captação de luz (Bulthuis 1983). A expansão dos rizomas verticais também é observada, visando a melhor exploração do ambiente e a utilização da irradiância subaquática disponível (Groth et al. 1996; van Tussenbroek 1996a).

Estimativas contínuas de parâmetros biométricos são importantes para quantificar os processos ecológicos de produção das espécies. Ambientes com

\footnotetext{
${ }^{1}$ Fundação Universidade Federal do Rio Grande, Departamento de Ciências Morfo-Biológicas, Av. Itália, km 8, Campus Carreiros, CEP 96201-900, Rio Grande, RS, Brasil

2 Fundação Universidade Federal do Rio Grande, Departamento de Oceanografia, Av. Itália, km 8, Campus Carreiros, CEP 96201-900, Rio Grande, RS, Brasil (uli@ecoscientia.com.br)

3 Autor para correspondência: dmbioni@furg.br
} 
alta turbidez, como o estuário da Lagoa dos Patos, dificultam a manipulação experimental em campo, fazendo-se necessária a realização de experimentos em laboratório. A importância desses estudos em laboratório está na possibilidade de manipular diferentes fatores e determinar seus efeitos sobre a fisiologia e a ecologia da espécie estudada (Harrison 1982; Schoschina 1996). Ruppia maritima desenvolve-se a partir da germinação de sementes, com ocupação de espaço por crescimento vegetativo (Dunton 1990), sendo de fácil cultivo e manutenção em condições de laboratório (Seeliger et al. 1984; Koch \& Dawes 1991).

Na maioria dos estuários, o crescimento, a distribuição e a produção das populações de $R$. maritima são controladas pela disponibilidade de luz, e sob irradiâncias reduzidas pode haver o desaparecimento de pradarias inteiras (Kantrud 1991). A transparência da água no estuário da Lagoa dos Patos tem comportamento sazonal, apresentando valores maiores de turbidez durante o inverno; processos de variação contínua, como ação de ventos e dinâmica de correntes, entretanto, também interferem na transparência da água (Niencheski \& Baumgarten 1997). Esses processos sazonais e diários modificam a transparência da água e, conseqüentemente, a irradiância subaquática, interferindo sobre o crescimento de populações subaquáticas como a da fanerógama submersa $R$. maritima (Seeliger 1997). Acredita-se que a espécie apresenta respostas demográficas diferenciadas, com capacidade de ajustar suas respostas modulares aos diferentes níveis disponíveis de irradiância subaquática. Dessa maneira, o objetivo desse estudo foi avaliar a influência da luz sobre o crescimento de $R$. maritima em cultivo experimental.

\section{Material e métodos}

Cultivo de $R$. maritima em laboratório - Sementes de Ruppia maritima L., coletadas em amostras de sedimento no estuário da Lagoa dos Patos, RS, Brasil, foram submetidas aos tratamentos de limpeza, indução de germinação e cultivo, segundo métodos estabelecidos por Seeliger et al. (1984). Plântulas recém germinadas, livres de epífitas, foram transportadas para frascos de cultivo (Fernbach), com volume de $1 \mathrm{~L}$, até produzirem biomassa suficiente para os experimentos. O meio de cultivo utilizado foi água de mar filtrada, autoclavada e enriquecida com solução nutritiva de von Stosch (von Stosch 1963), sem vitaminas (Seeliger et al. 1984). As plantas foram mantidas em sala de cultivo com temperatura de $20 \pm$
$1{ }^{\circ} \mathrm{C}$, salinidade $20 \%$, fotoperíodo de 12 horas luz / 12

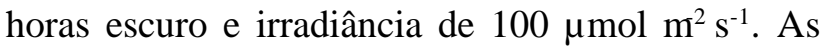
radiações utilizadas foram medidas com o aparelho LiCor Radiation Sensor LI-1000, usando como fontes de luz lâmpadas fluorescentes brancas frias, posicionadas sobre os frascos de cultivo. Para garantir nutrientes suficientes para o crescimento, o meio de cultivo foi trocado a cada três dias.

Crescimento de $R$. maritima em diferentes irradiâncias - Das plantas mantidas em estoque, foram selecionados cinco indivíduos, separados em seis repetições contendo um "ramet" cada (parte de rizoma, com nó, de onde partem 2-3 folhas e raízes). As repetições foram transferidas para frascos de cultivo (Fernbach) de 3L e expostas às irradiâncias de 40, 200 e $400 \mu \mathrm{mol} \mathrm{m}^{-2} \mathrm{~s}^{-1}$, correspondentes a $5 \%, 25 \%$ e $50 \%$, respectivamente, da irradiância incidente média anual no estuário da Lagoa dos Patos, estimada em $810 \mu \mathrm{mol} \mathrm{m} \mathrm{m}^{-2} \mathrm{~s}^{-1}$. Temperatura, salinidade, fotoperíodo e meio de cultivo foram os mesmos das condições do estoque. Após 10 dias de aclimatação, para cada nível de irradiância foram avaliados:

a) longevidade foliar, medida com base no tempo entre o início do experimento até a morte (folhas brancasamareladas) das primeiras quatro folhas produzidas em cada repetição, marcadas com caneta de tinta à prova d'água.

b) comprimento de folhas novas produzidas em cada repetição, marcadas com caneta de tinta à prova d'água, medido ao atingirem as idades de $6,12,18$, 24,30 e 40 dias. A seguir, as folhas foram levadas à estufa a $80^{\circ} \mathrm{C}$, para determinação da massa de matéria seca.

c) crescimento das plantas, medido diariamente, contando-se o número de folhas, raízes e novos "ramets" produzidos, durante no máximo 40 dias, para efeito de padronização. O crescimento, ao final deste período, foi também quantificado pelo número e comprimento de folhas, raízes e rizomas, bem como pelo número de ramificações do rizoma. $\mathrm{O}$ acréscimo de biomassa aérea (folhas) e subterrânea (rizomas e raízes) foi obtido a partir da massa de matéria seca.

A taxa de crescimento específico foi calculada pela equação: $\mu=\left(\ln B_{t}-\ln B_{o}\right) \cdot t^{1}$, onde: $B_{o}$ é a quantidade de biomassa inicial produzida e $\mathrm{B}_{\text {, a }}$ quantidade de biomassa final, após t dias de crescimento (Olesen \& Sand-Jensen 1993). A quantidade de biomassa inicial das folhas utilizadas nos experimentos foi estimada a partir da equação de regressão entre o comprimento foliar e a massa de matéria seca, de 50 folhas medidas independentemente. 
A taxa de crescimento específico foi expressa em é $\mathrm{g} \mathrm{d}^{-1} \mathrm{MS}$ (gramas por dia de matéria seca).

\section{Análises estatísticas}

Para as análises, foi utilizado o pacote estatístico STATISTICA para Windows 5.1 (SaltSoft, Inc. 1996). Inicialmente foi realizado um teste (ANOVA de duas vias) para verificar o efeito da intensidade luminosa sobre as respostas de crescimento das plantas. Como não foram observadas respostas diferenciadas, as plantas foram agrupadas em lotes por irradiâncias. ANOVA simples foi aplicada para testar a influência da irradiância sobre os parâmetros de crescimento de folhas, raízes e "ramets", após a normalização dessas variáveis pela expressão: raiz quadrada de x. Para o crescimento das folhas, em cada irradiância, utilizou-se o comprimento inicial da folha como co-variável, a ser descontada da análise. A relação entre a biomassa subterrânea: aérea foi feita por análises de Regressão Linear Simples, com os valores de biomassa normalizados pela expressão: $\log \mathrm{x}+1$. Todas as análises de variância foram seguidas de teste de comparação múltipla de Tukey a 5\% de significância (Zar 1984).

\section{Resultados e discussão}

Ruppia maritima tem sido considerada uma planta adaptada a ambientes com altas irradiâncias subaquáticas (Kantrud 1991). No presente estudo, plantas de $R$. maritima, mantidas em cultivo, apresentaram respostas de crescimento diferenciadas em função das irradiâncias fornecidas, com maior produção de folhas nas irradiâncias mais altas. A longevidade média das folhas em irradiância de $40 \mu \mathrm{mol} \mathrm{m}^{-2} \mathrm{~s}^{-1}$ (57,7 $\pm 2,7$ dias) foi maior do que nas irradiâncias de $200 \mu \mathrm{mol} \mathrm{m} \mathrm{m}^{-1}(38,1 \pm 1,8$ dias $) \mathrm{e}$ $400 \mu \mathrm{mol} \mathrm{m} \mathrm{m}^{-2} \mathrm{~s}^{-1}(35,1 \pm 1,6)$. Sabe-se que a longevidade das folhas de fanerógamas submersas depende das características ambientais, aumentando com a profundidade, o que representa uma medida indireta do decréscimo da irradiância subaquática (Pergent \& Pergent-Martini 1991). A longevidade das folhas pode estar também relacionada ao comprimento das folhas e com a taxa de renovação foliar, intrínsecas das espécies (Duarte 1991b). Populações de R. maritima, no estuário da Lagoa dos Patos, durante a estação de crescimento no verão, quando a irradiância subaquática é maior, apresentam rápido crescimento vegetativo, com grande investimento na produção de folhas (Seeliger 1997). A maior produção de folhas nas irradiâncias de 200 e $400 \mu \mathrm{mol} \mathrm{m} \mathrm{m}^{-1}$ e o prolongamento da longevidade das já existentes em irradiância de $40 \mu \mathrm{mol}$ $\mathrm{m}^{-2} \mathrm{~s}^{-1}$, sugerem modificações metabólicas similares às respostas demográficas da espécie no estuário da Lagoa dos Patos, durante a estação de crescimento no verão, quando a irradiância subaquática é maior.

Embora com menor produção diária de folhas, o comprimento final atingido pelas folhas foi maior em irradiância de $40 \mu \mathrm{mol} \mathrm{m} \mathrm{m}^{-2} \mathrm{~s}^{-1}$ (Tab. 1). O período de alongamento foliar médio foi de 24 dias, enquanto para as plantas que cresceram nas irradiâncias de $200 \mathrm{e}$ $400 \mu \mathrm{mol} \mathrm{m}^{-2} \mathrm{~s}^{-1}$, o crescimento foi estabilizado em 12 dias (Fig. 1). Medidas de comprimento das folhas são consideradas indicadores de estresse ambiental por luz, mostrando variações de acordo com a profundidade de ocorrência das fanerógamas submersas e com a época do ano. Maiores comprimentos são alcançados quando as condições de luminosidade são menores (van Tussenbroek 1996b; Lee \& Dunton 1997). No presente trabalho, em irradiância de $40 \mu \mathrm{mol} \mathrm{m} \mathrm{m}^{-2} \mathrm{~s}^{-1}$, a produção de folhas foi menor, mas o alongamento maior, indicando resposta fenotípica das plantas, visando a maior captação de luz. Já em irradiância de 200 e $400 \mu \mathrm{mol} \mathrm{m} \mathrm{m}^{-2} \mathrm{em}$ que o fator luz não foi limitante, a planta tendeu a investir em ocupação horizontal,

Tabela 1. Variáveis bióticas em plantas de Ruppia maritima em crescimento experimental durante 40 dias, sob diferentes irradiâncias. Produção diária de folhas, raízes e de novos "ramets", comprimento final de folhas, raízes e rizomas e número de ramificações dos rizomas.

\begin{tabular}{|c|c|c|c|c|c|c|c|}
\hline \multirow{2}{*}{$\begin{array}{l}\text { Irradiância } \\
\mu \mathrm{mol} \mathrm{m}{ }^{-2} \mathrm{~s}^{-1}\end{array}$} & \multicolumn{2}{|c|}{ Produção diária } & \multirow{2}{*}{$\begin{array}{c}\text { "Ramets" } \\
\text { totais } \\
\mathrm{n}^{\circ}\end{array}$} & \multicolumn{3}{|c|}{ Comprimento final } & \multirow{2}{*}{$\begin{array}{c}\text { Ramificações } \\
\text { do rizoma } \\
n^{\circ}\end{array}$} \\
\hline & $\begin{array}{l}\text { Folhas } \\
\mathrm{n}^{\text {o } \mathrm{dia}^{-1}}\end{array}$ & $\begin{array}{l}\text { Raízes } \\
\mathrm{n}^{\circ} \mathrm{dia}^{-1}\end{array}$ & & $\begin{array}{c}\text { Folhas } \\
\mathrm{cm}\end{array}$ & $\begin{array}{c}\text { Raízes } \\
\mathrm{cm}\end{array}$ & $\begin{array}{c}\text { Rizomas } \\
\mathrm{cm}\end{array}$ & \\
\hline 40 & $0,8 \pm 0,06^{\mathrm{a}}$ & $0,3 \pm 0,1^{\mathrm{a}}$ & $25,5 \pm 3,3^{\mathrm{a}}$ & $11,5 \pm 0,3^{\mathrm{a}}$ & $6,2 \pm 3,2^{\mathrm{a}}$ & $42,2 \pm 18,7^{\mathrm{a}}$ & $3,5 \pm 3,1^{\mathrm{a}}$ \\
\hline 200 & $1,9 \pm 0,2^{\mathrm{b}}$ & $0,6 \pm 0,4^{b}$ & $43,1 \pm 2,5^{\mathrm{b}}$ & $6,4 \pm 0,3^{\mathrm{b}}$ & $4,7 \pm 2,4^{b}$ & $51,3 \pm 14,8^{a}$ & $5,6 \pm 3,4^{b}$ \\
\hline 400 & $2,6 \pm 0,2^{c}$ & $0,8 \pm 0,4^{c}$ & $47,0 \pm 1,4^{\mathrm{c}}$ & $5,5 \pm 0,2^{c}$ & $3,8 \pm 2,1^{\mathrm{c}}$ & $52,4 \pm 20,8^{a}$ & $7,5 \pm 2,8^{c}$ \\
\hline
\end{tabular}

Médias nas colunas seguidas pela mesma letra não diferem estatisticamente $(P>5 \%)$ pelo teste de Tukey $(\mathrm{n}=30)$. 


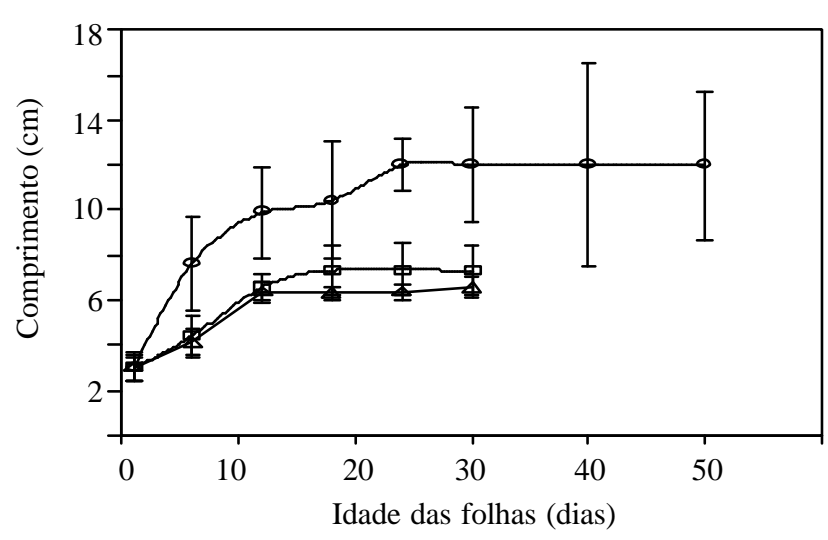

Figura 1. Comprimento das folhas de plantas de Ruppia maritima (médias \pm desvio padrão), em função de sua idade, sob diferentes irradiâncias: $40(\mathrm{O}), 200(\square), 400(\triangle) \mu \mathrm{mol} \mathrm{m}^{-2} \mathrm{~s}^{-1}$. (n = 80).

produzindo mais folhas, raízes e "ramets", que representam mais pontos de captação de luz e nutrientes, resultando em melhores condições de sobrevivência (Tab. 1).

Além da menor produção de folhas, foi observada também menor produção diária de raízes em irradiância $40 \mu \mathrm{mol} \mathrm{m} \mathrm{m}^{-2}$ (Tab. 1). A menor produção foliar pode resultar em redução na fotossíntese líquida das plantas. Sendo assim, o maior investimento na produção de tecidos não-fotossintetizantes, como as raízes, implicaria no aumento de custo metabólico para a manutenção das plantas, desviando o oxigênio produzido nas folhas para manter o metabolismo aeróbico das raízes (Caffrey \& Kemp 1991). A redução na produção de folhas, decorrente da diminuição da irradiância disponível, pode resultar na diminuição da produção de raízes e provocar um grande impacto na sobrevivência das plantas em ambientes muito instáveis (Zimmerman et al. 1989). A redução na produção de raízes em resposta à menor produção foliar, como observado, indica um mecanismo de compensação, evidenciando novamente a capacidade de adaptação de $R$. maritima às condições de luminosidade fornecidas.

O número de "ramets" produzidos também mostrou respostas diferenciadas em relação à irradiância (Tab. 1). Considerando que o "ramet" representa uma unidade ecológica primária capaz de manter vida independente da planta-mãe, quanto maior a sua produção, maior a propagação ou ocupação de espaço pela planta-mãe. Do ponto de vista demográfico, a produção de novos "ramets" representa melhor ocupação e exploração do ambiente, aumentando a probabilidade de sobrevivência. Do ponto de vista fisiológico, maior produção de novos "ramets" representa mais pontos de captação de luz e nutrientes (Groth et al. 1996; van Tussenbroek 1996a). Em condições ótimas de luminosidade e de oferta de nutrientes, as plantas tendem a apresentar crescimento horizontal, visando maior ocupação e exploração de espaço, enquanto em baixas irradiâncias, as plantas tendem ao crescimento vertical, visando melhor captação de luz (Pilon \& Santamaría 2002). A redução no crescimento, imposta pelas baixas irradiâncias subaquáticas, como observado, diminuindo a produção de folhas e de raízes e, conseqüentemente, o número de "ramets" formados, pode provocar grandes impactos nas populações de $R$. maritima, reduzindo a extensão de pradarias em ambientes com alta turbidez, como o estuário da Lagoa dos Patos.

O comprimento final alcançado pelos rizomas não mostrou diferenças significativas nas irradiâncias testadas, embora o número de ramificações dos rizomas tenha diferido em todas as irradiâncias (Tab. 1). Irradiâncias mais altas resultaram em maior produção foliar e maior número de ramificações. $\mathrm{O}$ incremento do número de ramificações dos rizomas pode diminuir a quantidade de irradiância que a planta pode interceptar devido ao auto-sombreamento, mas representa também um aumento na área de absorção de nutrientes (Haraguchi 1996). Considerando que não houve limitação externa de nutrientes, devido ao enriquecimento do meio de cultivo com os sais da solução de von Stosch, as diferenças observadas no número de ramificações foram, provavelmente, devidas às variações nas irradiâncias em que as plantas cresceram. Nas irradiâncias mais elevadas, uma vez que a luz não representou fator limitante, as plantas passaram a investir em expansão horizontal, produzindo mais ramificações. Resultados similares foram encontrados em Thalassia testudinum e Zostera noltii, em que o incremento nas ramificações foi diretamente relacionado ao incremento na irradiância disponível (Tomasko \& Lapointe 1991; Vermaat \& Verhagen 1996).

As massas individuais das folhas de plantas crescendo em irradiância de $40 \mu \mathrm{mol} \mathrm{m} \mathrm{m}^{2} \mathrm{~s}^{-1}$ foram significativamente maiores. Apesar da maior massa individual e maior alongamento foliar, o número total de folhas produzidas foi menor, resultando em menor biomassa total (Tab. 2, Fig. 2). O mesmo ocorreu com os valores de biomassa aérea, biomassa subterrânea e relação biomassa subterrânea / biomassa aérea, que foram significativamente menores nessa irradiância (Tab. 2). A relação entre a biomassa subterrânea / biomassa aérea pode ser 
Tabela 2. Biomassa da parte aérea (PA), subterrânea (PS) e biomassa total, relação biomassa parte subterrânea / aérea (PS/PA) e taxa de crescimento específico das folhas de Ruppia maritima, crescendo durante 40 dias em cultivo experimental sob diferentes irradiâncias. $(\mathrm{n}=30)$.

\begin{tabular}{|c|c|c|c|c|c|}
\hline \multirow{2}{*}{$\begin{array}{l}\text { Irradiância } \\
\mu \mathrm{molm}^{-2} \mathrm{~s}^{-1}\end{array}$} & \multicolumn{3}{|c|}{ Biomassa } & \multirow[t]{2}{*}{ PS/PA } & \multirow{2}{*}{$\begin{array}{c}\text { Taxa de crescimento } \\
\text { específico } \\
\left(\mathrm{g} \mathrm{dia}^{-1}\right)\end{array}$} \\
\hline & $\begin{array}{l}\text { Aérea } \\
\text { (g de MS) }\end{array}$ & $\begin{array}{l}\text { Subterrânea } \\
\text { (g de MS) }\end{array}$ & $\begin{array}{c}\text { Total } \\
\text { (g de MS) }\end{array}$ & & \\
\hline 40 & $0,06 \pm 0,07^{\mathrm{a}}$ & $0,03 \pm 0,04^{\mathrm{a}}$ & $0,09 \pm 0,10^{\mathrm{a}}$ & $0,59 \pm 0,04^{\mathrm{a}}$ & $0,07 \pm 0,04^{\mathrm{a}}$ \\
\hline 200 & $0,09 \pm 0,06^{\mathrm{b}}$ & $0,08 \pm 0,07^{\mathrm{b}}$ & $0,17 \pm 0,13^{\mathrm{b}}$ & $0,92 \pm 0,06^{\mathrm{b}}$ & $0,11 \pm 0,05^{\mathrm{b}}$ \\
\hline 400 & $0,08 \pm 0,04^{b}$ & $0,08 \pm 0,06^{\mathrm{b}}$ & $0,17 \pm 0,10^{\mathrm{b}}$ & $0,96 \pm 0,07^{\mathrm{b}}$ & $0,12 \pm 0,06^{\mathrm{b}}$ \\
\hline
\end{tabular}

Médias nas colunas seguidas pela mesma letra não diferem estatisticamente $(P>5 \%)$ pelo teste de Tukey $\quad(\mathrm{n}=30)$.

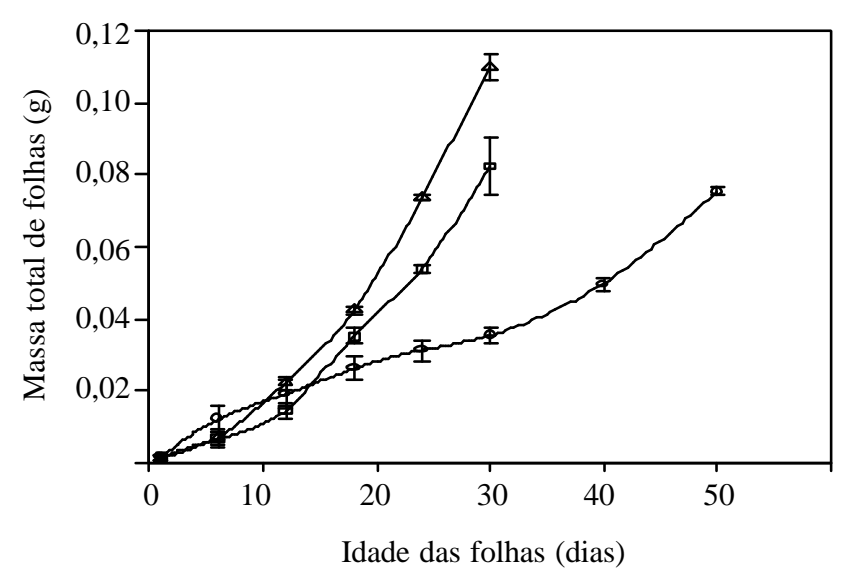

Figura 2. Massa total de folhas de Ruppia maritima (médias \pm desvio padrão), em plantas crescendo sob diferentes irradiâncias: 40 (O), $200(\square), 400(\triangle) \mu \mathrm{mol} \mathrm{m} \mathrm{m}^{-2} \mathrm{~s}^{-1}$. (n = 30).

crítica para o crescimento em ambientes com intensidade reduzida de luz (Fourqurean \& Zieman 1991). A biomassa aérea de $R$. maritima foi diferentemente afetada pela irradiância, assim como a biomassa subterrânea, evidenciando uma alocação de recursos para o incremento do alongamento foliar e redução da massa dos tecidos nãofotossintetizantes. Resultados similares foram observados, durante os picos de desenvolvimento das pradarias dessa espécie, no estuário da Lagoa dos Patos, onde a maior alocação de biomassa pelos caules floríferos e folhas, durante a estação de crescimento, foram relacionados aos níveis mais altos de irradiância subaquática e aos períodos de floração (Costa \& Seeliger 1989; Silva \& Asmus 2001).

As taxas de crescimento específico das folhas de R. maritima, medidas pelas variações na biomassa aérea, foram maiores nas irradiâncias de $200 \mathrm{e}$ $400 \mu \mathrm{mol} \mathrm{m} \mathrm{m}^{-2} \mathrm{~s}^{-1}$ (Tab. 2), evidenciando, mais uma vez, a adaptação da espécie a ambientes com alta luminosidade. Esses resultados foram coincidentes com os observados em outras fanerógamas submersas como, por exemplo, Zostera marina (Zimmerman et al. 1989; Olesen \& Sand-Jensen 1993) e Ruppia drepanensis (Santamaría \& Hootsmans 1998), cujas taxas de crescimento específico também foram afetadas por fatores abióticos e foram maiores em irradiâncias subaquáticas mais elevadas.

Ruppia maritima tem, como característica demográfica, uma rápida produção e morte antecipada dos "ramets", após a produção de sementes (Silva \& Asmus 2001). No presente trabalho, não foi observada a floração, nem a produção de sementes. Embora as maiores irradiâncias tenham propiciado maior produção, nenhuma das irradiâncias testadas impediu o crescimento e o desenvolvimento vegetativo da espécie. Em conclusão, esse estudo, dá suporte à hipótese de que $R$. maritima pode se ajustar a diferentes condições de luminosidade, modificando suas características demográficas e respostas de crescimento, o que lhe permite ocupar hábitats com níveis variáveis de irradiância subaquática, como no estuário da Lagoa dos Patos.

\section{Agradecimentos}

Os Autores agradecem ao Programa de Doutorado em Oceanografia Biológica da Fundação Universidade Federal do Rio Grande (FURG), pelo apoio técnico e à Coordenação de Aperfeiçoamento de Pessoal de Nível Superior (Capes), pelo apoio financeiro.

\section{Referências bibliográficas}

Bulthuis, D.A. 1983. Effects of in situ light reduction on density and growth of the seagrass Heterozostera tasmanica (Martens ex Aschers.) den Hartog in western port, Victoria, Australia. Journal of Experimental Marine Biology and Ecology 67: 91-103. 
Caffrey, J.M. \& Kemp, W.M. 1991. Seasonal and spatial patterns of oxygen production, respiration and rootrhizome release inPotamogeton perfoliatus and Zostera marina L. Aquatic Botany 40: 109-128.

Carruthers, T.J.B.; Walker, D.I. \& Kendrick, G.A. 1999. Abundance of Ruppia megacarpa Mason in a seasonally variable estuary. Estuarine, Coastal and Shelf Science 48: 497-509.

Costa, C.S.B. \& Seeliger, U. 1989. Vertical distribution and resource allocation of Ruppia maritima L. in southern Brazilian estuary. Aquatic Botany 33: 123-129.

Duarte, C.M. 1991a. Seagrass depth limits. Aquatic Botany 40: $363-377$.

Duarte, C.M.1991b. Allometric scaling of seagrass form and productivity. Marine Ecology Progress Series 77 : 289-300.

Dunton, K.H. 1990. Production ecology of Ruppiamaritima L. s.l. and Halodule wrightii Aschers. in two subtropical estuaries. Journal of Experimental Marine Biology and Ecology 143: 147-164.

Fourqurean, J.W. \& Zieman, J.C. 1991.Photosynthesis, respiration and whole plant carbon budget of the seagrass Thalassia testudinum. Marine Ecology Progress Series 69: 161-170.

Groth, A.T.; Lovett-Doust, L. \& Lovett-Doust, J. 1996. Population density and module demography in Trapa natans (Trapaceae), an annual, clonal aquatic macrophyte. American Journal of Botany 83: 1406-1415.

Haraguchi, A. 1996. Rhizome growth of Menyanthes trifoliata L. in a population on a floating peat mat in Mizorogaike Pond, central Japan. Aquatic Botany 53: 163-173.

Harrison, P.G. 1982. Seasonal and year-to-year variations in mixed intertidal populations of Zostera japonica Aschers. and Graebn. and Ruppia maritima L. s.l. Aquatic Botany 14: 357-371.

Kantrud, H.A. 1991. Widgeon grass (Ruppia maritima L.): a literature review. Fishing Wildlife Research 10: 1-58.

Koch, E.W. \& Dawes, C.J. 1991. Ecotypic differentiation in populations of Ruppia maritima L. germinated from seeds and cultured under algae-free laboratory conditions. Journal of Experimental Marine Biology and Ecology 152: 145-159.

Lee, K. \& Dunton, K.H. 1997. Effects of in situ light reduction on the maintenance, growth and partitioning of carbon resources in Thalassia testudinum Banks ex König. Journal of Experimental Marine Biology and Ecology 210: 53-73.

Menéndez, M. 2002. Net production of Ruppia cirrhosa in the Ebro Delta. Aquatic Botany 73: 107-113.

Niencheski, L.F. \& Baumgarten, M.G. 1997. Química Ambiental. Pp. 21-25. In: U. Seeliger; C. Odebrecht \& J.P. Castello (eds.). Subtropical convergence environments. The coast and sea in the Southwestern Atlantic. Springer-Verlag Berlin Heidelberg.

Olesen, B. \& Sand-Jensen, K. 1993. Seasonal acclimatization of eelgrass Zostera marina growth to light. Marine Ecology Progress Series 94: 91-99.
Pergent, G. \& Pergent-Martini, C. 1991. Leaf renewal cycle and primary production of Posidonia oceanica in the bay of Lacco Ameno (Ischia, Italy) using lepidochronological analysis. Aquatic Botany 42: 49-66.

Pilon, P. \& Santamaría, L. 2002. Clonal variation in morphological and physiological responses to irradiance and photoperiod for the aquatic angiosperm Potamogeton pectinatus. Journal of Ecology 90: 859-870.

Santamaría, L. \& Hootsmans, M.J.M. 1998. The effect of temperature on the photosynthesis, growth and reproduction of a Mediterranean submerged macrophyta, Ruppia drepanensis. Aquatic Botany 60: 169-188.

Schoschina, E.V. 1996. Seasonal and age dynamics of growth and reproduction of Phycodrys rubens (Rhodophyta) in the Barents and White Seas. Aquatic Botany 55: 13-30.

Seeliger, U. 1997. Submersed spermatophytes. Pp. 27-30. In: U. Seeliger; C. Odebrecht \& J.P. Castello (eds.). Subtropical convergence environments. The coast and sea in the southwestern Atlantic. Springer-Verlag Berlin Heidelberg.

Seeliger, U.; Cordazzo, C. \& Koch, E.W. 1984. Germination and algal-free laboratory culture of widgeon grass, Ruppia maritima. Estuaries 7: 176-178.

Silva, E.T. \& Asmus, M.L. 2001. A dynamic simulation model of the widgeon grass Ruppia maritima and its epiphytes in the estuary of the Patos Lagoon, RS, Brazil.Ecological Modelling 137: 161-179.

Tomasko, D.A. \& Lapointe, B.E. 1991. Productivity and biomass of Thalassia testudinum as related to water column nutrient availability and epiphyte levels: field observations and experimental studies. Marine Ecology Progress Series 75: 9-17.

van Tussenbroek, B.I. 1996a. Integrated growth patterns of turtle grass, Thalassia testudinum Banks ex König. Aquatic Botany 55: 139-144.

van Tussenbroek, B.I. 1996b Leaf dimensions of transplants of Thalassia testudinum in a Mexican Caribbean reef lagoon. Aquatic Botany 55: 133-138.

Vermaat, J.E. \& Verhagen, F.C.A. 1996. Seasonal variation in the intertidal seagrassZostera noltii Hornem.: coupling demographic and physiological patterns. Aquatic Botany 52: 259-281.

von Stosch, H.A. 1963. Wirkung von Arsenit auf Meeresalgen in Kultur. Pp. 142-150. In: Proceeding of the 4th International Seaweed Symposium. Biarritz, A.D. Virville, J. Feldmann Ed.

Zar, J.H. 1984. Biostatistical Analysis. 2 $2^{\mathrm{a}}$ ed. New Jersey, Prentice-Hall, Inc.

Zimmerman, R.C.; Smith, R.D. \& Alberte, R.S. 1989. Thermal acclimation and whole-plant carbon balance in Zostera marina L. (eelgrass). Journal of Experimental Marine Biology and Ecology 130: 93-109. 